\title{
Escritura, vida y convivencia en Motivos de Proteo de José Enrique Rodó
}

\section{Writing, life and coexistence in Motivos de Proteo by José Enrique Rodó}

\section{Ottmar Ette*}

Resumen: En este artículo se hace un análisis de la obra de José Enrique Rodó, Motivos de Proteo, en función de discusiones teóricas contemporáneas. El libro del uruguayo funciona para analizar los fenómenos de la literatura y un Saber Sobre Vivir; en él se manifiesta una escritura fragmentaria y archipiélica organizada bajo una perspectiva polilógica. Esta estética y epistemología del texto contiene, al mismo tiempo, un saber de la vida y de la convivencia.

Palabras clave: José Enrique Rodó, Escritura polilógica, Saber Sobre Vivir.

ABstract: This article analyses the work by José Enrique Rodó Motivos de Proteo according to contemporary theoretical discussions. The Uruguayan's book is a useful tool for analising literary phenomena and a knowledge about living. It portrays a fragmentary and archipelagic writing organised under a polilogic perspective. At the same time, this text's aesthetics and epistemology contains a knowledge of coexistence.

Key words: José Enrique Rodó, Polilogic thinking, Knowledge about Living

Recibido: 20 de febrero de 2018

Aceptado: 4 de abril de 2018

Universidad de Postdam, Alemania (ette@uni-postdam.de). 


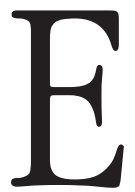

n uno de sus textos quizás más vanguardistas e inquietantes, su ensayo "Der Erzähler, Betrachtungen zum Werk Nikolai Lesskows" de 1936 Walter Benjamin ya había puesto de relieve cuán difícil se había convertido el narrar sobre el entorno (2007: 103128). Sus reflexiones son por momentos una premonición de aquella paulatina desaparición del narrar, que sin lugar a dudas estamos viviendo y tenemos que lamentar en la actual sociedad del facebook (Simanowski 2016). Porque la pérdida que sufriremos sin el narrador y el narrar, lo supo plasmar Benjamin en el último párrafo de su ensayo a través de estas bien elegidas palabras:

Así considerado, el narrador es admitido junto al maestro y al sabio. Sabe consejos, pero no para algunos casos como el proverbio, sino para muchos, como el sabio. Y ello, porque le está dado recurrir a toda una vida. (Por lo demás, una vida que no sólo incorpora la propia experiencia, sino, en no pequeña medida, también la ajena. En el narrador, lo sabido de oídas se acomoda junto a lo más suyo.) Su talento es de poder narrar su vida y su dignidad; la totalidad de su vida. El narrador es el hombre que permite que las suaves llamas de su narración consuman por completo la mecha de su vida. En ello radica la incomparable atmósfera que rodea al narrador, tanto en Lesskow como en Hauff, en Poe como en Stevenson. El narrador es la figura en la que el justo se encuentra consigo mismo (128).

El narrar, podríamos añadirle a estas palabras, siempre apunta hacia una totalidad. Quiere y apunta hacia, revive y nos muestra, ofrece y nos regala una totalidad continua compleja: la vida completa como un saber acerca de la vida, que también es un saber de vida, un saber vivenciar, un saber sobre/vivir, un saber convivir y un saber trans/vivir (Ette [2004]-2010; Ette 2007: 7-32; Asholt y Ette 2010; Ette y Ugalde 2015). En la literatura no se trata de una mímesis artística en forma de una realidad representada, sino de una representación de realidades vividas (aunque no necesariamente por uno mismo). 
En el narrar se condensa la miniaturización modelar y el anhelado deseo de reunir todo el mundo en un libro, en un solo texto. En esas mismas miniaturizaciones, las literaturas del mundo se despliegan como Fractales del mundo (Ette 2017), un afán que ya se percibe en los giros de Walter Benjamin: cuando en la literatura se trata de abrazar la totalidad, esto es, la vida. El narrador es siempre más que un simple narrador, porque la literatura es siempre más de lo que es. Desde todos sus diferentes inicios nos narra acerca de las formas y las normas de vida en el mundo y en todos aquellos mundos del pasado, del presente y del futuro que siempre deben considerarse en plural —como pasados, como presentes y sobre todo como futuros. Incluso en su giro hacia la memoria, como recuerdo del pasado, la literatura es ante todo y siempre esto: es prospectiva. Pero, ¿de qué forma podemos narrar la totalidad de la vida, el todo del mundo? Y, ¿cómo se ve el saber de las literaturas del mundo, en el que Benjamin no solamente reconocía un saber específico, sino descubría el centelleo de un fragmento de sabiduría?

Ya en el primer capítulo de la obra maestra del romanista Erich Auerbach, Mimesis. Dargestellte Wirklichkeit in der abendländischen Literatur, elaborada entre mayo de 1942 y abril de 1945 durante su exilio en Estambul y quien mantenía una relación epistolar conmovedora con Walter Benjamin, había reconocido en Occidente dos líneas de tradiciones no solo diferentes entre sí, sino también diferenciables entre ellas para hacer irradiar todo el mundo en su totalidad. Así, el primer modelo fundamental sería "el texto narrativo bíblico" que apunta, según el comentario sagaz pero muchas veces ignorado de Auerbach, hacia la representación abarcadora de la "historia mundial; comienza con el inicio del tiempo, con el génesis y terminará con el momento final, cuando se cumpla la profecía gracias a la que el mundo encontrará su final" (1982: 18). No hay nada imaginable allende este modelo del mundo: todo tiene que referirse a él -solo a él.

El segundo modelo elemental de una comprensión abarcadora del mundo lo conforman en Occidente, según Auerbach, los "poemas homéricos": ellos determinan "cierta relación de acontecimientos limitados tanto en el tiempo como en el espacio" (18). Al lado de ellos es posible 
que haya otras contextualidades $-\mathrm{y}$ por ende, otras narraciones que se pueden idear tanto teórica como artísticamente, sin que por ello entren en conflicto con el diseño del mundo homérico. Junto al mundo de Homero pueden existir otros mundos (como mundos narrativos).

Así, el modelo del mundo bíblico del Antiguo Testamento está "compuesto más ostensiblemente de piezas sueltas", pero estas "piezas individuales" pertenecen, a diferencia de los poemas homéricos, "todos a una relación histórico-mundial y de interpretación de la historia mundial" (19). Por consiguiente, le corresponde a la fragmentariedad espacio-temporal limitada de la Ilíada y de la Odisea una gran unidad narrativa, mientras que la "perspectiva religiosa e histórico-mundial" (19) unificada del Antiguo Testamento aparece a nivel del texto en una fragmentariedad compuesta de piezas. Se podría hablar aquí de una dinámica narrativa bipolar, que impulsa e impele la literatura occidental en su conjunto de una forma paradójica como un motor de dos tiempos. Hay - y esto no debe sorprendernos - indicios de que la doble línea de creación de mundos en Occidente puesta de relieve por Auerbach también puede encontrarse en formas relevantes de expresión de otras latitudes histórico-culturales y teórico-culturales.

Que se hayan podido dar y desarrollar, entre las líneas tradicionales aquí diferenciadas en el nivel de las literaturas del mundo en Occidente, siempre las más diferentes mezclas se sobreentiende, también, en aras del personaje que aquí se presentará -esto es, en las creaciones literarias del modernista uruguayo José Enrique Rodó. En tendencia se puede constatar que la línea de tradición continua que nombramos como primera, tiende más bien hacia una miniaturización cuantitativamente menor del modelo aspirado que la tradición fractal, que se orienta en formas de expresión insulares y más breves; una tradición que contiene en su forma diminutiva por lo menos una miniatura más del modelo, en forma de una abreviación de una página, de un párrafo o de una sola frase: son artefactos literarios que se han convertido en objeto de estudio de la nanofilología (Ette 2008; Rueda 2017). Éste también es un aspecto que se tratará en las siguientes reflexiones. 
Si desde el canto épico del Gilgamesh en Mesopotamia y el Shi-Jing en China, esto es, desde los tan diferentes comienzos de las literaturas del mundo, se logran identificar ambos modelos narrativos en las más diversas culturas, lenguas, temporalidades y espacialidades (Ette 2017), entonces se puede afirmar con miras a las formas literarias breves - que siempre jugaron un papel primordial en el proceso creativo de José Enrique Rodó (Rodríguez Monegal 1967: 19-139; Suiffet 1995; Ette y Heydenreich 2000)-, que éstas se encuentran en la línea de tradición de un diseño con cuya ayuda se desenvuelve experimentalmente una fuerza estética demiúrgica (Menke 2008) que logra su objetivo valiéndose en esencia de la archipielización y la fractalización. ${ }^{1}$ Aquí hay que tomar en consideración que, gracias a la copresencia de los más diferentes textos cortos que no necesariamente tienen que estar dispuestos como fragmentos, un modo fractal y archipiélico de la narración, puede dar a luz un juego de relacionalidad que pone en el centro de todo el texto la cotextualidad, esto es, la presencia o convivencia conjunta de todos los textos cortos dentro de una forma mayor.

De allí resulta muchas veces una relación de reciprocidad entre cotextualidad y contextualidad que rebasa en mucho los correspondientes contextos históricos y políticos y también literarios y culturales. El juego de la cotextualidad con los más diversos contextos devela estéticamente el modelo de una lógica relacional en la que todo se intercambia con todo. Cada texto-isla funciona en este caso en un doble sentido, como una isla-mundo independiente, pero al mismo tiempo conforma, gracias a la relacionalidad de todos los elementos, un mundo-isla, en tanto mundo de islas, porque todas las islas funcionan como en un archipiélago por sí solas y en interacción, en su auto-lógica y en su conectividad. La pluralidad de islas y mundos de islas no es en este sentido una simple suma de las estructuras, sino una relacionalidad de estructuraciones complejas y abiertas.

1 Sobre aspectos formales y cuestiones de estilo en Motivos de Proteo, véanse Ibáñez (1967: 7-53) y Real de Azúa (1957: 7-138). 
Es precisamente en las formas literarias cortas y ultracortas donde hay una intersección entre miniatura y modelo, de lo erótico-sensual con la teoría en una conformación experimental que se despliega a partir de una poética del movimiento y que por ende reclama para su análisis conceptos de una poética vectorial, profundamente dinámica y asimismo fundada en la nanofilología. La elección y el diseño estético de una forma de tal índole ya se puede entender en sí como un alegato en favor de modelos polilógicos de comprehensión que se sustraen a cualquier tipo de estatismo y construyen su modelo en constante movimiento, como cambio permanente. No puede contemplarse aquí desde una tranquilidad estable: en una obra así, todo se encuentra en perpetuo movimiento que es asimismo convivencia (Ette 2012).

Con una forma en movimiento comienza el primero de los 158 capítulos o "motivos", contenidos en el volumen Motivos de Proteo. ${ }^{2}$ publicado por vez primera en abril de 1909:

Reformarse es vivir... Y desde luego, nuestra transformación personal en cierto grado, ino es ley constante e infalible en el tiempo? ¿Qué importa que el deseo y la voluntad queden en un punto si el tiempo pasa y nos lleva? El tiempo es el sumo innovador. Su potestad, bajo la cual cabe todo lo creado, se ejerce de manera tan segura y continua sobre las almas como sobre las cosas. Cada pensamiento de tu mente, cada movimiento de tu sensibilidad, cada determinación de tu albedrío, y aún más: cada instante de la aparente tregua de indiferencia o de sueño, con que se interrumpe el proceso de tu actividad consciente, pero no el de aquella otra que se desenvuelve en ti sin participación de tu voluntad y sin conocimiento de ti mismo, son un impulso más en el sentido de una modificación, cuyos pasos acumulados producen esas transformaciones visibles de edad en edad, de decenio en decenio; mudas de alma, que sorprenden acaso a quien no ha tenido ante los ojos el gradual desenvolvimiento de una vida, como sorprende al viajero que torna, tras larga ausencia, a la patria, ver las cabezas blancas de aquellos a quienes dejó en la mocedad (Rodó 1967: 309).

2 En cuanto a esta obra opacada hasta la actualidad por el Ariel de Rodó, véanse Rodríguez Monegal (1967: 301-308) y Real de Azúa (1976: ix-xxxv). En cuanto a la complejidad del corpus, tomando en consideración los Motivos de Proteo nuevos y novísimos que aquí no se profundizarán, véase, entre otros, Real de Azúa (1948: 91). 
En este incipit, en el primer párrafo del primer motivo del texto de enumeración romana, cuya brevedad varía y cuya duración de lectura es de entre un minuto y más o menos diez, aparecen los lexemas, temas y motivos elementales de todo el volumen. El público lector podía distinguir desde el inicio de los Motivos de Proteo que en las relaciones de la figura del título, Proteo y el viejo y venerable maestro, Próspero en Ariel de Rodó, ${ }^{3}$ prevalecía esa consonancia entre el yo y el mundo, entre las edades de la vida y del tiempo, entre los impulsos literarios y filosóficos y se extendían hasta las repeticiones sonoras y la igualdad silábica de ambas figuras. No cabe duda que el escritor uruguayo había construido a propósito esas ostensibles paralelas entre las voces de Proteo y Próspero para sus lectores, porque sabía muy bien que en la historia de la recepción de su tan famosa obra de 1900, la voz de su Próspero se equiparaba con la suya e incluso (aunque falsamente) se había confundido con la suya (Barbagelata 1920: 24). Sin embargo, aunque aparece una vez más la figura del Maestro, esta nueva obra no es una continuación de la anterior.

No obstante, en los Motivos de Proteo — como ya lo mostraba el incipit- tenemos una estructura oracional paratáctica de gran valor artístico que es puesta en cadencia gracias a la introducción de frases cortas. José Martí ya había creado una forma escritural de cuño paratáctico complejo, en la que las largas ondas de sus oraciones eran interrumpidas por estructuras oracionales breves como si fueran rompientes que generaban un ritmo que puede considerarse característico en la prosa del Modernismo hispanoamericano (Ette 1994a: 9-58). José Enrique Rodó había seguido de forma muy sagaz el desarrollo de esta prosa modernista, pese a que es poco común encontrar en la lengua castellana frases tan largas y muchas veces difíciles de entender a primera vista y en el Ariel todo culminó en formas de expresión complejas y altamente estetizantes, lo que elevó la prosa finisecular a un grado de complejidad insuperable (García 1992: 75-88; Gutiérrez 1987: 495-506; Meyer-Minnemann 1979; Achugar 1994:

3 Sobre el Ariel de Rodó véanse Brotherston 1967: 1-19; Castro 1995: 125-161; Castro 2000: 9-128. 
233-256). El rebuscamiento de la sintaxis de Rodó presenta un obstáculo en la recepción y al parecer fue un gran desafío para más de un traductor. Tal y como ya sucediera en su Ariel, en el que José Enrique Rodó se había servido de los más diversos géneros - desde el discurso académico y político, pasando por narraciones intercaladas hasta el ensayo literario o el tratado científico (Ette 1994b: 193-240), este gran maestro de la prosa breve practica una combinación de diversas tradiciones genéricas. Los más diferentes textos y tipos de texto conviven aquí. Entre los géneros incluidos e integrados en este volumen publicado en 1909 se encuentran tanto el aforismo como el estudio de caracteres, la confesión autobiográfica, el ensayo didáctico, el cuento fantástico, el diálogo filosófico, el ejemplo, el fragmento, la parábola, el poema en prosa, la prédica moralizante, el cuadro literario, el discurso político-filosófico o el tratado psicológico, sin olvidar su práctica de la cita explícita e implícita. En un texto dilucidador de 1908, en el que concluye su trabajo en los Motivos, que posteriormente se incluyó en El Mirador de Próspero -lo que una vez más subraya la cercanía entre Próspero y Proteo- Rodó acentuaba que en última instancia se movía en un terreno intermedio entre la ciencia y el arte (533). Aquí, donde de mil formas se podían combinar entre sí la verdad y la belleza, el pensamiento moderno había creado "obras intermedias, singularmente adecuadas a nuestro gusto y a nuestras necesidades espirituales" (533). Con sus Motivos de Proteo presentaba una "obra intermedia".

Sin lugar a dudas, Rodó se había movido ya en su Ariel en ese espacio intermedio entre verdad y belleza, arte y ciencia. Ahora quería dejar atrás su obra que tanto éxito había tenido para presentar nueve años después un proyecto literario-filosófico más ambicioso aún, en tanto los Motivos de Proteo no solamente son el libro más voluminoso sino también aquel en el que el espíritu formador y reformador del autor uruguayo se presentó en formas de expresión y sendas mucho más radicales. Los Motivos son un libro experimental.

Ante este telón de fondo, volvamos una vez más al incipit de los Motivos de Proteo. La frase introductoria que ha sido interpretada sobre todo desde un enfoque individual y psicológico, "Reformarse es vivir..." 
(1967: 309) y que aparece una y otra vez con diferentes modificaciones en el transcurso de ese escrito a manera de un leitmotif, pone la forma, la reforma, la reformación y la reformulación en relación con la vida que se puede considerar como en perpetuo movimiento y renovación. Se le confiere al lexema vida en este volumen de 1909 un significado superior. Lexemas como movimiento, transformación, innovación o impulso subrayan además el hecho de que en la vida nada puede permanecer en estado inmóvil, y de que en el "gradual desenvolvimiento de una vida" (310) en el sentido metafórico (y goetheano) de un desenvolver (Entwickelung), en el que la única constante es el cambio.

La estructuración abierta se podría comparar en el nivel teórico-artístico, literario y cultural quizás más adecuadamente con las concepciones de Umberto Eco, que recién medio siglo después se concretizaron en su Opera aperta (1962) y Struttura assente (1968). Si además buscamos desarrollos literarios que se pudieran poner en relación con este tipo de técnica ordenadora, entonces habría un ostensible vínculo con la novela de Julio Cortázar, Rayuela, publicada en 1963, a la que subyace un juego de brincos y saltos y con ello la posibilidad de leer los capítulos según su numeración o según secuencias fijadas de antemano (o absolutamente libre según un recorrido de lectura elegido por el mismo lector) (Ette 2009a: 197-228). En este momento podríamos indicar que José Enrique Rodó se movió en un terreno de praxis literaria y teórico-literaria que no nació bajo el signo del Modernismo, sino bajo el de una estética posmoderna que comenzó a dominar en los años sesenta del siglo XX. Son evidentes un sinnúmero de relaciones transversales entre el Modernismo hispanoamericano y una estética posmoderna que se internacionalizó rápidamente, pero que nunca se puede pensar sin Jorge Luis Borges.

Si contemplamos con mayor detenimiento los paratextos de sorprendente complejidad que encabezan los Motivos de Proteo, entonces se perfilan más referencias entre el Modernismo tardío del escritor uruguayo y ciertos desarrollos literario-estéticos de la segunda mitad del siglo pasado. Así, Rodó le antepuso a sus Motivos, inmediatamente después de una cita extraída del Evangelio de San Marcos (IV, 11: "Todo se trata por 
parábolas"), el siguiente texto corto firmado con sus iniciales que, por su importancia, se transcribirá en toda su extensión:

No publico una "primera parte" de PROTEO: el material que he apartado para estos Motivos da, en compendio, idea general de la obra, harto extensa (aun si la limitase a lo que tengo escrito) para ser editada de una vez. Los claros de este volumen serán el contenido del siguiente; y así en los sucesivos. Y nunca PROTEO se publicará de otro modo que de éste; es decir, nunca le daré "arquitectura" concreta, ni término forzoso; siempre podrá seguir desenvolviéndose, "viviendo". La índole del libro (si tal puede llamársele) consiente, en torno de un pensamiento capital, tan vasta ramificación de ideas y motivos, que nada se opone a que haga de él lo que quiero que sea: un libro en perpetuo "devenir", un libro abierto sobre una perspectiva indefinida (Rodó 1967: 308).

Aquí hallamos la metafórica del "desenvolvimiento" y una vez más se habla de la vida. El carácter vivo y en este contexto abierto de este libro que difícilmente se puede considerar como libro en el sentido tradicional, consiste en remitir una y otra vez a tomos nuevos, partes nuevas, pensamientos nuevos dentro de aquella moderna literatura de ideas y este libro nunca terminado, nunca concluido es su renovada concretización. Ninguna arquitectura fija debe fungir como camisa de fuerza para esta voluntad absoluta a una apertura perspectivista. Se trata por lo tanto de un libro profundamente experimental que desafía explícitamente las fronteras de la escritura de libros, para crear aquella "ramificación de ideas y motivos" (309), que sin lugar a dudas podremos denominar una interconexión y también un rizoma (Deleuze y Guattari 1976) de la propia escritura. La vuelta final de este paratexto no solo habla del libro abierto sino, más aún, de un "libro en perpetuo devenir" (309).

Además, su intención - tal y como él mismo lo formulara- era la de abarcar "todo lo creado" (309) en el sentido que le diera en su incipit. Que en este caso se trata de una relación profunda entre la forma literaria breve y el deseo de capturar toda la creación, toda la vida; que hay una relación entre la forma microtextual y la pretensión macrológica, entre el microcosmos de los textos breves y el macrocosmos de todo lo creado en 
el sentido etimológico de "cosmos" y con ello de la configuración de orden y belleza, se debería de haber puesto de relieve ya en la primera parte de esta nueva aproximación al fascinante texto y modelo de comprehensión de Rodó. Los Motivos de Proteo de Rodó conforman un libro con vida en incesante devenir (y por eso probablemente tan difícil de comprender).

Pero, ¿de qué manera se podrían describir con más exactitud las ligas y relaciones entre los dos lexemas de la sentencia introductoria - esto es, forma y vida? ¿Cómo se podría desarrollar a partir de allí una nueva comprensión de la dimensión narrativa, por medio de la que se podrían vincular más íntimamente la forma y la reforma por un lado, y por el otro, la vida y el revivir?

La pregunta por la vida en los Motivos de Proteo implica seguramente la pregunta por la vida misma de Rodó, esto es, por elementos autobiográficos que el gran estilista uruguayo sin lugar a dudas entremezcló en sus motivos. Sin embargo, al usar el lexema de la vida, no solo quería hablar de su vida o de una vida, sino de la vida toda: de un saber de la vida en la vida y para la vida: en consecuencia, por un saber de vida, que se abre también hacia las dimensiones del saber vivenciar, del saber sobrevivir, del saber convivir y del saber transvivir. ¿De qué manera, empero, se puede reflexionar sobre la vida toda y, en tanto forma, poder llevarla a cabo desde el punto de vista literario-estético?

Desde el inicio de los Motivos de Proteo, la vida se encuentra bajo el signo del tiempo y, con ello, de constantes cambios que incluyen también y sobre todo las transformaciones sicológicas en la vejez. Estas transformaciones sicológicas jugaron un papel temático muy importante en una carta que Rodó le dirigiera a Miguel de Unamuno el 12 de octubre de 1900 (1967: 1379; Ette 2009a: 197-227). En sus reflexiones literarias, Rodó intentaba sobre todo poner de relieve el incesante trabajo en el propio yo y en esta trayectoria encontraba una y otra vez nuevos ejemplos, con lo que 
se ocupó en el transcurso de sus Motivos, con gran ímpetu experimental y también con mucho empeño, en la búsqueda y recolección de éstos. Sin lugar a dudas: el ímpetu experimental del escritor se apoyaba en una ética de trabajo de las dimensiones de un Flaubert.

La red quizás más estable y segura que apoya la construcción de los Motivos está conformada de biografías y biografemas ejemplares. Entre los modelos que tratan la relación de cambio entre reformarse y vivir se encuentra Johann Wolfgang von Goethe, quien es citado en numerosas ocasiones a lo largo de los Motivos y a quien Rodó le confiere un papel casi paradigmático en él.

El más alto, perfecto y típico ejemplar de vida progresiva, gobernada por un principio de constante renovación y de aprendizaje infatigable, que nos ofrezca, en lo moderno, la historia natural de los espíritus, es, sin duda, el de Goethe. Ninguna alma más cambiante que aquélla, vasta como el mar y como él libérrima e incoercible; ninguna más rica en formas múltiples; pero esta perpetua inquietud y diversidad, lejos de ser movimiento vano, dispersión estéril, son el hercúleo trabajo de engrandecimiento y perfección, de una naturaleza dotada, en mayor grado que otra alguna, de la aptitud del cultivo propio; son obra viva en la empresa de originar lo que él llamaba, con majestuosa imagen, la pirámide de mi existencia (1967: 406).

En esta exaltación de Goethe por parte de Rodó, que culmina en el giro final de esta introducción al motivo, no solamente se resalta el papel extraordinario que jugaba el creador del Fausto para el escritor de los Motivos de Proteo, sino asimismo el significado de este ejemplo para la relación de tensión entre los lexemas de forma y vida, que aquí una vez más se pone de relieve. Si todavía en el Ariel eran de primordial importancia los espacios de enseñanza del Maestro (González 1985: 21), o las esferas ligeras de Ariel, el airy spirit que Rodó se prestara de Shakespeare, mientras que el mar, el Caribe, habían sido marginados, juegan un papel fundamental bajo el signo del archipiélago, del mundo de islas griego de Proteo en los textos proteicos de Rodó. Porque aun antes del primer capítulo, Rodó introduce, inmediatamente después del título "Proteo", en la segunda edición de sus Motivos, la deidad proveniente de la mitología 
griega de la siguiente manera - y este párrafo es mucho más que solo un ejercicio didáctico para facilitar la comprensión de la obra:

Forma del mar, numen del mar, de cuyo seno inquieto sacó la antigüedad fecunda generación de mitos, Proteo era quien guardaba los rebaños de focas de Poseidón. En la Odisea y en las Geórgicas se canta de su ancianidad venerable, de su paso sobre la onda en raudo coche marino. Como todas las divinidades de las aguas, tenía el don profético y el conocimiento cabal de lo presente y lo pasado. Pero era avaro de su saber, esquivo a las consultas, y para eludir la curiosidad de los hombres apelaba a su maravillosa facultad de transfigurarse en mil formas diversas (309).

Recurrir a la Antigüedad grecorromana aun ejemplar para Rodó (representada por Homero y Virgilio y la deidad marítima ambigua, Proteo) sirve al escritor de Montevideo para convertir las diferentes visiones del mar en la superficie lúdica de sus textos y en el paisaje de la teoría, en el que todo se encuentra en movimiento, fluye y cambia. Numen y figura del dios y sus incesantes transfiguraciones conforman la personificación de la vida como constante cambio, como forma en devenir, en un reformarse sin cesar, como una vida en versátil transfiguración. Justamente en este momento, la figura de Goethe obtiene su espacio de movimiento proteico:

No hay en su vida sol que ilumine la imitación maquinal, el desfallecido reflejo, de lo que alumbraron los otros. Cada día es un renuevo de originalidad para él. Cada día distinto; cada uno de ellos, consagrado, como un Sísifo de su propia persona, a levantar otro Goethe de las profundidades de su alma, nunca cesa de atormentarle el pensamiento de que dejará la concepción de su destino incompleta: ambicionaría mirar por los ojos de todos, reproducir en su interior la infinita complejidad del drama humano, identificarse con cuanto tiene ser, sumergirse en las mismas fuentes de la vida... Llega así al pináculo de su ancianidad gloriosa, aún más capaz y abierta que sus verdes años, y expira pidiendo más luz, y este anhelo sublime es como el sello estampado en su existencia y su genio, porque traduce, a la vez, el ansia de saber en que perseveró su espíritu insaciable, y la necesidad de expansión que acicateó su vitalidad inmensa... (407). 
Johann Wolfgang von Goethe se convierte aquí en un caso modelar y ejemplo de una vida que se renueva sin cesar, creando así una sucesión de otros Goethe que impresionan menos por su alteridad que por la constante dinámica de expansión (su necesidad de expansión). El concepto entero de los Motivos de Proteo no apunta a la creación de simples alteridades, sino a una epistemología de la expansión (Ette 2016: 331-355), que no descansa en la producción maquinal de oposiciones. Porque alteridad se convierte en peligro mortal allí, donde su producción se enfoca hacia la elaboración de superioridades y sobre todo inferioridades.

José Enrique Rodó no se somete a aquella lógica fatal y peligrosa que puede reconocerse desde la Conquista en cuanto a la cuestión de lo otro que objetó Tzvetan Todorov (1982) y en parte también Julia Kristeva (1991), aunque más bien referida a la Modernidad- y esto me parece ser un principio que diferencia sustancialmente los Motivos de Proteo del concepto de oposiciones que predomina en el Ariel; un principio que va de la mano con la forma elegida por el autor. El pensamiento esquiva una tradición de pensar en alteridades, que actualmente se considera peligrosa y que ha acuñado la per-cepción y actuación de los europeos desde el inicio de la conquista del continente americano por las potencias ibéricas a más tardar desde finales del siglo XV y la filosofía europea incluso hasta en la actualidad (Descombes 1981). Más bien hace el intento de pensar una ampliación y una dilatación por ejemplo de subjetividad y vida en un contexto de patrones de pensamiento relacional que van ganando terreno a las alteridades discursivas, de tal manera que de ahí se pueda derivar y desarrollar una verdadera epistemología de la ampliación.

Lo cual no significa que haya superado del todo las estructuras bipolares del pensamiento, tal y como se perfilan en su concepción americanista de un continente dividido en dos, en un norte utilitarista y un sur latino y cultivado (Ardao 1977: 14); no obstante, se delinea un potencial de desarrollo en el pensamiento y, más aún, en la escritura archipiélica de José Enrique Rodó que se abren hacia patrones de percepción y reflexión polilógicos. Se puede perfilar entre otros en el cuadro de la poliperspectividad, del deseo de la figura de Goethe creada por Rodó, de poder mirar 
a través de los ojos de todos o de la complejidad infinita de la vida humana que no solamente destella en este momento, sino que ya desde las frases iniciales pone de relieve la necesaria re-formación de todo ser, de toda vida. Las tantas veces repetidas palabras finales de Goethe, repetidas también por Lezama Lima en La expresión americana, apuntan a un más, apuntan hacia aquella ampliación que culminan al final de este pasaje en las palabras de "a necesidad de expansión" (Rodó 1967: 407) y pueden pensarse a la par con la vivacidad, la vitalidad. Todo eso permite un nuevo pensar ampliado (weiteresDenken).

En este momento se muestran con contundencia las motivaciones y con ello, los Motivos, pero en última instancia también las consecuencias que acompañan la decisión de Rodó en pro de una escritura profundamente relacional y archipiélica. Los fenómenos evocados y representados no solo se incluyen en cadenas causales o consecutivas, sino que aparecen en zonas de imbricación compleja, en las que todo está relacionado con todo -aunque no siempre simultáneamente. Una escritura de tal índole es capaz de crear, precisamente por la valorización de lo interrumpido, de lo discontinuo y, sobre todo, de lo fractal, un cuadro estéticamente convincente de toda la vida desde un enfoque multiperspectivístico y no una sola vida en la vida de un (destacado) individuo. Nada en los Motivos de Proteo es independiente.

Entre las múltiples repeticiones de esta frase clave "Reformarse es vivir", que pueden rastrearse a lo largo de los Motivos de Proteo, cuenta también la introducción al motivo ochenta y seis, donde el primer párrafo dice lo siguiente: "La práctica de la idea de nuestra renovación tiene un precepto máximo: el viajar. Reformarse es vivir. Viajar es reformarse" (412). Una vez más, Rodó recurre a una fórmula definitoria breve.

Con estas frases y sentencias que se logran grabar en la memoria y de alguna manera recuerdan las estructuras oracionales discursivas y rítmicas de José Martí, Rodó se abre el camino de una relación de reciprocidad bipolar a una tripolar, en la que en un nivel explícito, la recurrencia al lexema pone en el centro de atención el reformarse. Asimismo, en el nivel implícito se le asigna al lector la tarea de cerrar el triángulo semán- 
tico por un "viajar es vivir" o un "vivir es viajar". El tópico del viaje de la vida que una y otra vez se manifiesta en los más diferentes biografremas y autobiografemas en los Motivos de Proteo, no debería tener mayor peso que la equiparación paradigmática del viaje con la vida. Si se contempla la biografía de José Enrique Rodó en la actualidad, la de un hombre que viajó mucho menos que los otros dos grandes modernistas, entonces lo paradójico de esta lección, de esta doxa radica en última instancia en que precisamente su único viaje transatlántico fuera el que lo llevara a Europa como corresponsal, a escribir esa última palabra (dolore) y a la muerte a Palermo.

Poco sorprende que la figura del viajero sea una altamente recurrente y con multiplicidad de imbricaciones aparece una y otra vez en los Motivos de Proteo. Pese a esto hay también en este ámbito una figuración paradigmática que no lleva el nombre de Goethe, sino el de Alexander von Humboldt, porque también el nombre del creador del Cosmos surca los Motivos de Proteo del escritor uruguayo de forma recurrente. Allí, no solo se menciona la extraordinaria longitud de la vida de este viajero (y con ello, la relación directa entre vivir y viajar), quien murió a los 89 años en 1859 en Berlín, esto es, medio siglo antes de la publicación de los Motivos, sino sobre todo la increíble amplitud y profundidad de los acervos del saber que el "Viejo de la Montaña", tal y como se le tildaba de broma, había recopilado y combinado. El nombre de Alexander von Humboldt es per se símbolo de la combinatoria y las posibilidades de combinación.

A pesar de que parte de los estudios biográficos en la actualidad suelen caer en una representación unívoca del científico natural y callan los no menos grandiosos logros del sabio prusiano como investigador de las culturas antiguas de América, de las lenguas americanas, como historiador o como teórico de la globalización, Rodó no cae en la trampa del inadmisible unilateralismo y se pregunta, no solo con miras a Humboldt, acerca de los vínculos trascendentales del saber. Así, podemos leer en el capítulo 108:

La observación del mundo material tiene por objeto abstraer las leyes generales a que obedecen las cosas y los seres, de donde nace la sabiduría del físico, del químico y del biólogo; o bien, estudiar concretamente las 
cosas y los seres mismos, describiéndolos y caracterizándolos, como hacen el geógrafo y el naturalista. Estos distintos sentidos de la observación se relacionan entre sí de modo que ninguno puede considerarse en absoluto ajeno de los otros; y sus relaciones objetivas se reproducen, a menudo, subjetivamente, en la vocación y la aptitud del sabio. El geógrafo naturalista, favorecido en ambos respectos por la facultad de aproximar dos órdenes de hechos tan fundamentalmente vinculados, se personificaría en la gran figura de Humboldt (1967: 445).

Tanto los estudios del volcán Pichincha, las investigaciones botánicas, el análisis de las lenguas indígenas, de los espacios, tiempos y culturas efectuados por Humboldt, le convierten en una figura y, más aún, en la figuración de un saber abarcador que surge de la combinatoria de los más diversos ámbitos de saber especializado. Este saber del sabio no se estanca en el saber especializado, sino que se unifica para conformar aquella sabiduría que José Enrique Rodó sin lugar a dudas admiró tanto en el Cosmos. En esta suma de la ciencia de Humboldt, el erudito desarrolló su gran meta, de unir en un solo libro el mundo en su totalidad. Así, le confesó a su amigo Varnhagen von Ense en una carta fechada el 24 de octubre de 1834 llena de esa euforia escéptica característica en él:

Ich fange den Druck meines Werks (des Werks meines Lebens) an. Ich habe den tollen Einfall, die ganze materielle Welt, alles was wir heute von den Erscheinungen der Himmelsräume und des Erdenlebens, von den Nebelsternen bis zur Geographie der Moose auf den Granitfelsen, wissen, alles in Einem Werke darzustellen, und in einem Werke, das zugleich in lebendiger Sprache anregt und das Gemüth ergötzt. Jede große und wichtige Idee, die irgendwo aufgeglimmt, muß neben den Thatsachen hier verzeichnet sein (1860: 20).

Los paralelismos con el proyecto de José Enrique Rodó son obvios. El escritor y prosista uruguayo también habría podido considerar sus Motivos de Proteo como la obra de su vida, ya que resumía como ninguna de sus obras sus incursiones a diferentes ámbitos del saber, sus vastas lecturas así como su forma de escribir en la escritura corta. A pesar de no dedicarse con tanto empeño al mundo material como lo hiciera Humboldt, 
confluían en una sola obra de su "moderna literatura de ideas" (1967: 229) todas las ideas de envergadura. Una convivencia de objetos y discursos dispares era de mayor importancia para ambos escritores.

Si tomamos en consideración que la formulación de la literatura de ideas de Rodó le debe mucho a la lectura intensa de los escritos de Friedrich Nietzsche y sobre todo su Así babló Zaratustra (Ette 1994a, b, c), se logra reconocer que aquí la idea de la totalidad de alguien como Humbol$\mathrm{dt}$, de representar el mundo (e incluso todo el cosmos) en la totalidad, se llevó a cabo en los Motivos de Proteo con ayuda de una técnica literario-filosófica que desarrollara Friedrich Nietzsche en sus escritos. Los Motivos de Proteo conforman así un Cosmos en escritura corta nietzscheana, producida por las más diversas hojas acerca de las más diferentes ideas organizadas según el modelo nietzscheano tanto en su aislamiento de isla como en su relacionalidad hasta conformar un todo vivo, que en el sentido de la palabra vive de la multiplicidad de posibilidades de combinación. El "Así habló Zaratustra" de Nietzsche y la respuesta americana de Rodó, el "Así habló Próspero", aún no habían perdido su resonancia tan poco tiempo después del fin de siglo. Los Motivos querían ser un libro de la vida toda.

\section{MOTIVOS DE PROTEO O LA VISIÓN DE LA CONVIVENCIA EN UN MUNDO TOTAL}

Si en la parte anterior se hizo hincapié en la importancia de escritores y filósofos del ámbito de lengua alemana en el pensamiento de Rodó, es cierto que muchos trabajos (incluidos los propios) han hablado de la dominación de referencias europeas y específicamente francesas en la obra completa del escritor uruguayo. Así, Carlos Real de Azúa por ejemplo, efectuó un conteo de los más diversos autores que aparecen en el texto, clasificados según el país de origen, su profesión, el número de menciones y otros criterios. En vista de un análisis estadístico de tal índole no deberíamos perder de vista que los Motivos de Proteo, similar a Ariel, no solamente 
disponen de un imbricado de alusiones explícitas, sino también implícitas de igual importancia. No obstante, es interesante tomar en consideración que entre los autores aludidos en los Motivos se encuentran a lo largo de los siglos 20 griegos, 19 españoles, 8 alemanes, 4 norteamericanos y no menos de 71 franceses (1976: 69).

El espacio literario explícito, esto es, la biblioteca que Rodó conforma en su libro, contiene ejemplares predominantemente europeos y está dominada por una imbricación helenista -francesa que revela el greco-galicismo mencionado por Rodó. Las escasas referencias y alusiones a autores hispanoamericanos son casi siempre de naturaleza implícita (Ette 1994b: 193-240). La biblioteca de Rodó se orienta en el concepto de una literatura mundial dominada por Europa y no en el concepto de la naciente perspectiva de las literaturas del mundo. La asimetría de las relaciones siguieron vigentes en el intercambio trasatlántico aun en las postrimerías del siglo XX.

La figura del autor, del escritor, del artista para Rodó sin lugar a dudas - tal y como lo revelan muchos de sus motivos- es la de un viajero. En los Motivos de Proteo de Rodó el movimiento de viaje escenifica al mismo tiempo el movimiento interior del alma (como en la literatura de viajes de la mística española), el movimiento hermenéutico del lector (quien, como compañero de diálogo es parte de la interlocución y cuya capacidad de lectura se pone constantemente a prueba) y el movimiento creativo del escritor por el universo de los objetos dados y ya existentes (Ette 2009a: 69). ¿No se compara el gran artista con Dios quien, como demiurgo crea el mundo y ve que ha quedado bien? (Rodó 1967: 421)

Es significativo además que la omnipresencia del viajero en estos Motivos toma en consideración las fases de globalización acelerada y trata de orientarse en las figuras de "viajeros europeos". En este sentido, la figura de Cristóbal Colón tiene una importancia paradigmática para el autor uruguayo -así como para Alexander von Humboldt, cuya madre hugonote no en balde se apellidaba Colomb. Como la verdadera figura de aceleración de la primera fase de globalización acelerada, Colón es símbolo del viajero como descubridor y colonizador, al mismo tiempo también -en el sentido 
que le diera Tzvetan Todorov- es el hermeneuta e interpretador de signos, quien entiende el mundo como un libro abierto y quien comienza a leer en el libro de la naturaleza de América.

Pero también se intercalan en los Motivos de Proteo viajeros de otras fases de globalización acelerada. Recurriendo al famoso capítulo acerca de los "Anregungsmittel zum Naturstudium" en el segundo tomo del Cosmos de Humboldt (2004: 189-239), Rodó desarrolla en el capítulo veinticinco de sus Motivos, la motivación para viajar, para el estudio de la naturaleza y para la descripción poética, tomando en consideración al trotamundos James Cook y otros viajeros y lo hace de la siguiente manera:

El espectáculo del mar visto por primera vez; un árbol que cautiva la atención, por hermoso o por extraño, son sensaciones que han experimentado muchos sin que nada de nota se siguiese a ellas; pero la primera visión del mar fue para Cook, y luego para aquella mujer extraordinaria, amazona de empresas pacíficas, que se llamó Ida Pfeiffer, la revelación de su genial instinto de viajeros; y Humboldt nos refiere en el Cosmos cómo de una palma de abanico y un dragonero colosal, que vio, de niño, en el jardín botánico de Berlín, partió el precoz anuncio del anhelo inextinguible que le llevó a conocer tierras remotas (1967: 369).

La vienesa Ida Pfeiffer, sin lugar a dudas la representante más famosa y espectacular de viajes de mujeres circunnavegantes en el siglo XIX, se encuentra aquí a la par con James Cook y Alexander von Humboldt, quienes con sus viajes marcan la segunda fase de globalización acelerada y es símbolo de la relación triangular de viajar, reformarse y vivir o con ello de un concepto de vida que se refiere a la vida toda en un mundo total por el que viajan, cruzándolo y circunnavegándolo. Esta interacción de una vida completa con un mundo total, la combinatoria de los más diversos ámbitos del saber en una obra que el mismo Rodó hubiera podido considerar la obra de su vida, así como la continua inscripción de su propia figura en este desarrollo por él perfilado: todo esto caracteriza los Motivos de Proteo, que -de forma similar al Cosmos para Humboldt- representan algo así como la suma de toda una vida, lo cual es más que una vida individual. El saber acerca de cada vez más vidas imbrica y amplía el conocimiento 
de la vida en la vida: el narrador Rodó incluye, en el sentido de Walter Benjamin, las vidas ajenas en la propia y amplía así los fundamentos del propio saber de vida.

A diferencia de Alexander von Humboldt, quien en sus escritos se convirtiera en el primer teórico de la globalización (Ette 2009b), José Enrique Rodó fue un pensador de la globalidad, cuyo interés no era tanto auscultar los contextos históricos de las transformaciones, sino más bien las transfiguraciones de las imbricaciones globales: José Enrique Rodó se comprendía a sí mismo y su propia vida como parte de esta globalidad allende las fronteras nacionales. Esta conciencia de mundo de Rodó sale a flote en aquellos textos dramáticos y a la vez reflexionados en los que se ocupa del final de la tercera fase de globalización acelerada, cuando ésta se consume en el fuego de la Primera Guerra Mundial y desaparece abruptamente. A pesar de que Rodó no pudo, a causa de su sorpresiva muerte en 1917, vivir el fin de este primer gran incendio mundial del siglo xx y la ulterior barbarie de la civilización y pese a que no pudiera dar un informe acerca de su último viaje justamente a una Europa carcomida por la guerra, sus reflexiones documentan una conciencia de mundo que se sabe en el intercambio transatlántico. Veía la América como continente integrada de forma especial en esta globalidad.

Aunque nunca puso en duda su lealtad hacia su Francia en este conflicto devastador, cuyas dimensiones poco a poco se perfilaban con más nitidez y combatía en el Río de la Plata todo lo que estuviera a favor de Alemania, en tanto valía la máxima -según un artículo salido de su pluma el 3 de septiembre de 1914- "La causa de Francia es la causa de la humanidad" (1967: 1220-1222), también hacía hincapié en un artículo del 9 de agosto de 1914 escrito para el Diario del Plata, que el mundo desde hace mucho se había convertido en un solo organismo. Pero, iqué significa una guerra en un organismo único y unido? En el último artículo que acabamos de mencionar, Rodó hablaba, bajo el título "Ansiedad Universal", de una solidaridad más que nunca indispensable:

Apenas hay lugar en el espíritu público para otra atención y otro interés que los que despierta la contienda que interrumpe el orden de la vida 
civilizada en los más cultos y poderosos pueblos del mundo. La solidaridad humana se pone a prueba en estos extraordinarios momentos, manifestando hasta qué punto la frecuencia y facilidad de las comunicaciones han hecho del planeta entero un solo organismo cuyos centros directores transmiten a los más apartados extremos la repercusión moral y material de lo que en ellos pasa (1218).

Esta convicción de que nuestro planeta se había convertido en una unidad viva gracias a los nuevos medios de comunicación del telégrafo y el cable transoceánicos, aboceta el cuadro e incluso la visión de una globalidad bajo el signo de una civilización que, a causa del conflicto bélico perceptible en todo el mundo, se había interrumpido y sin lugar a dudas, estaba amenazada. A causa de esto se cuestionaban fundamentalmente las formas y normas de convivencia existentes.

José Enrique Rodó retomó aquí en 1914 una visión planetaria que había desarrollado - sin la sombra de una futura guerra- en el capítulo ochenta y dos de sus Motivos de Proteo:

Este inmenso organismo moral que del mundo, para nuestros abuelos dividido en almas nacionales, como en islas del archipiélago, han hecho la comunicación constante y fácil, el intercambio de ideas, la tolerancia religiosa, la curiosidad cosmopolita, el hilo de telégrafo, la nave de vapor, nos envuelve en una red de solicitaciones continuas y cambiantes. [...] Ninguna edad como la nuestra ha comprendido el alma de las civilizaciones que pasaron y la ha evocado a nueva vida, valiéndose de la taumaturgia de la imaginación y el sentimiento; y por este medio también, el pasado es para nosotros un magnetizador capaz de imponernos sugestiones hondas y tenaces, no limitadas ya, como cuando el entusiasmo histórico del Renacimiento, al legado y el genio de una sola civilización, sino procedentes de dondequiera que la humanidad ha perseguido un objetivo ideal y volcado en troquel nuevo y enérgico su espíritu (408).

En este párrafo se muestra el nuevo espíritu de un nuevo Renacimiento, que no se orienta más en una sola civilización, sino (por lo menos potencialmente), en muchas. Lo que en aquel entonces existía en aislamiento y separación en islas individuales y casi sin comunicación entre sí, ahora ha sido incluido en un proceso de intercambio que ha creado 
una unidad viva y cada vez más unificada. La vida se desarrolla así a escala mundial y gana intensidad, porque al mismo tiempo adquiere profundidad histórica y se abre hacia un futuro planetario común: la multiplicidad de combinaciones que se extiende a lo largo y ancho del mundo vincula todo transarchipiélicamente.

En esta retrospectiva al siglo pasado y su devenir, los Motivos de Proteo ocupan una postura abierta y casi entusiasta hacia el futuro, similar a la del saludo dinámico al nuevo siglo en el Ariel. Sin embargo, a diferencia de Ariel, los Motivos de Proteo han sido concebidos hacia una ampliación que remite al espacio y al tiempo, pero se orienta sobre todo en el sinnúmero de civilizaciones y en la vida, cuyas formas y normas ya no se desarrollan en un sentido monocultural. También aquí se trata de una convivencia. Corresponde sin lugar a dudas a una epistemología de la ampliación, que modificó de manera fundamental el pensamiento de Rodó y lo preparó a pensar un desarrollo global fundamentalmente cambiado y más abierto.

En los Motivos de Proteo, Rodó no desarrolló ninguna visión del futuro para América Latina, sino una perspectiva para todo el mundo -aunque solo visto desde el Mirador de Próspero. El lugar del sabio Maestro Próspero lo ocupaba ahora el dios griego, en cuyas transformaciones no debía aparece solamente una vida, sino la vida toda en un mundo global. No había aquí ninguna visión que se refiriera a América Latina -y esta carencia podría ser la explicación para el hecho de que los Motivos de Proteo nunca encontraron la entusiasta resonancia de Ariel- pero una visión de un mundo del mañana bajo el signo de una convivencia archipiélica. Se trata aquí, como hemos podido ver, de un texto de estructura compleja que ha sido elaborado bajo el signo de la convivencia de géneros narrativos y no-narrativos: del texto de una moderna literatura de las ideas, que - como antaño Humboldt- trata de conjuntar todo un cosmos de ideas. No es la vida de unos cuantos protagonistas, sino la vida de un sinnúmero de figuras la que se entreteje aquí, pero los biografemas de todas estas figurae vitae no se unen para formar una biografía, sino para conformar un acervo de saber de vida. 
Para la convivencia de los géneros, de las ideas y de las vidas, el modernista uruguayo encontró la forma móvil de textos breves y ultra breves que mantienen su propia lógica, su auto-lógica, tal y como sucede con las islas. Sin embargo, también entran en una múltiple relación archipiélica y transarchipiélica, que podría describirse como aquella de los diferentes archipiélagos de uno y el mismo mundo. Con ello el aislamiento de antaño entre islas y archipiélagos pertenece al pasado: todo está vinculado con todo, todo es intercambio recíproco.

Sin duda, los Motivos se pueden relacionar con diferentes momentos temporales, continentales y de lenguas; conforman series que pueden clasificarse por sus campos temáticos, como por ejemplo cambio y transformación, tiempo y transitoriedad, arte y cultura, literatura y escritura, desarrollo individual y sociedad, viajes y viajeros, religión y fe, voluntad y poder, amor y amistad o agonía y muerte. Sin embargo, ninguna de estas series puede reclamar para sí ser el punto neurálgico, "central", superior a todos los demás.

En esta obra, lo que se debe considerar esencial o marginal no se ha sometido a ninguna jerarquía, a ninguna centralización. Todas estas diferentes lógicas valen al mismo tiempo, y más: no son solo equivalentes, sino equipolentes, porque no únicamente disponen de la misma validez, sino también del mismo poder. La igualdad de poder de estas series discontinuas hace surgir un espacio de movimiento de las imbricaciones, por lo que se produce una multirrelacionalidad abierta, polilógica. Quizás éste es el cuadro más valioso, la visión más impresionante de una convivencia de las lógicas, que José Enrique Rodó quiso ofrecer a su público lector por medio de Proteo. Los Motivos de Proteo de José Enrique Rodó por lo tanto tuvieron que quedar inconclusos y más aún: eran categóricamente inconcluibles, en tanto los textos cortos y ultracortos, las nuevas hojas y notas no se agregaban sino que se añadían: en la finitud y el finiquito de un libro se encuentra la infinitud y falta de conclusión de la forma fractal.

Antes del capítulo final muy breve, que se abre en primera persona del singular a "mi nuevo sentir", "mi nueva verdad", "mi nueva palabra" 
(495), Rodó sitúa un cuadro conmovedor y móvil, que concluye y abre sus Motivos de Proteo de una forma muy diferente a la de Ariel:

Sentado a la ventana, empleo mi ocio en la contemplación. Mientras en mi chimenea se abre un ojo de cíclope que desde hace tiempo permanecía velado por su párpado negro, y junto a mí mi galgo ofrece sus orejas frías y sedosas a las caricias de su amo, se fija mi atención en una muda sinfonía: la de las hojas que, desprendidas, en bandadas sin orden de los árboles que van dejando desnudos, pueblan el suelo y el aire, a la merced del viento. Me intereso, como en una ficción sentimental, en sus aciagas aventuras. Ora se alzan y van en el vuelo loco; ora, más al abrigo, ruedan solitarias breve trecho y quedan un momento inmóviles, antes de trazar, lánguidamente, otro surco; ora se acumulan y aprietan, como medrosas o aterridas; ya se despedazan y entregan en suicidio a la ráfaga, deshechas en liviano polvo; ya giran sin compás alrededor de sí mismas, como poseídas danzantes... Su suerte varia es pasto de mi fantasía, cosquilleo de mi corazón. Me parecen en ocasiones los despojos volantes de un sacrificio de papeles viejos, con los que se avientan cartas de amores idos y vanidades de la imaginación, obras que no pasaron de su larva (494).

La muda sinfonía de las hojas se transforma, bajo la mirada del yo contemplativo, en una coreografía de hojas que representan un perpetuum mobile en siempre nuevas posiciones y figuras, movimientos y diseminaciones que no es dominada por ninguna jerarquía, por ningún orden. Lo único inderogable es el movimiento. En configuraciones cada vez nuevas, las hojas entablan siempre nuevas relaciones, se unen para formar constelaciones transitorias, efímeras y representan probablemente un baile, un baile de muerte. Cada hoja es independiente y se encuentra a la vez en una relación múltiple con las otras que no existirían sin cada una de las hojas individuales. De una manera estéticamente muy bien lograda, un yo narrador es capaz de develar la visión abierta y también viva de la estructuración de una obra, que no se deja fijar, no se deja inhibir en sus movimientos. La visión de las hojas es la mise en abyme de todo el conjunto textual móvil de las hojas individuales. No hay nada al final de este libro que encuentre reposo, cese. Todo se mantiene en un movimiento que tampoco se detiene en el momento de la muerte, sino que apunta hacia la trascendencia. Aun en la muerte trasluce la vida, en el baile de la muerte se perfila el saber 
transvivir que tendrán estas hojas a pesar de que aquellos que han escrito esta historia y sus historias ya no se encuentran entre nosotros. El libro mismo se mantiene vivo. Las hojas no figuran la finitud y transitoriedad. Transfiguran su propia convivencia en una transvivencia, que se abre hacia lo inconcluso, lo interminable y, por ende, a un pedacito de infinidad en su estado puro.

Es muy probable que la figura del narrador personificado en este yo sumergido en la contemplación y, más aún, en esta visión concluyente de los Motivos de Proteo ya no nos sea familiar y quizás inaccesible. En las primeras líneas del ensayo referido más arriba, Walter Benjamin remarcó: "El narrador - por muy familiar que nos parezca el nombre- no se nos presenta en toda su incidencia viva. Es algo que de entrada está alejado de nosotros y que continúa a alejarse aún más" (2007: 103). Sin duda: aquí estamos confrontados con un narrador en el sentido que Benjamin le diera en la cita introductoria; un narrador que engulle lo ajeno para convertirlo en parte de su propia vida y lo transforma en una vida completa, en la vida misma: enfardando un saber y muchos saberes más. Al enfardar la vida se vuelve accesible estéticamente como saber de vida y se transfiere a una multirrelacionalidad polilógica de una estructuración abierta, móvil.

Así surge al final de las muchas vidas aquello que Walter Benjamin, en su intento por explicar el narrador y el narrar, denominara sabiduría. Pero, ¿somos capaces de encontrar la voz de esta sabiduría, de este saber in-disciplinado, en la voz del narrador? El narrador se dirige a nosotros como sabio: atravesando los espacios y los tiempos, siempre con la esperanza de no estar demasiado lejos, apartado, de sernos ajeno. Nosotros podemos volver a percibir hoy esta voz que nos es tan familiar de una manera inquietantemente acogedora.

BIBLIOGRAFÍA

ACHúGAR, HugO. "Fin de siglo. Reflexiones desde la periferia". Posmodernidad en la periferia. Enfoques latinoamericanos de la nueva teo- 
ría cultural. Hermann Herlinghaus y Monika Walter editores. Berlin: Langer Verlag, 1994. 233-256.

ARDAO, ARTURO. "Prólogo: El americanismo de Rodó". José Enrique Rodó: La América Nuestra. Arturo Ardao compilación y prólogo. La Habana: Casa de las Américas 1977. 8-23.

ASHOlt, Wolfgang y OTTMAR ETTE. Literaturwissenschaft als Lebenswissenschaft. Programm - Projekte - Perspektiven. Tübingen: Gunter Narr Verlag, 2010.

AUERBACH, ERICH. Mimesis. Dargestellte Wirklichkeit in der abendländischen Literatur. Bern - München: Francke Verlag, 1982.

Barbagelata, Hugo D. "A manera de Prólogo". Rodó y sus críticos. París: Imprenta de Mr. Vertongen, 1920.

Benjamin, WaLter. "Der Erzähler. Betrachtungen zum Werk Nikolai Lesskows". Erzäblen. Schriften zur Theorie der Narration und zur literarischen Prosa. Ausgewählt und mit einem Nachwort von Alexander Honold. Frankfurt am Main: Suhrkamp, 2007. 103-128.

BROTHERSTON, GORDON. "Introduction". José Enrique Rodó. Ariel. Gordon Brotherston edición, introducción y notas. Cambridge: Cambridge University Press, 1967. 1-19

Castro Morales, BelÉn. "Un proyecto intelectual en la encrucijada de la modernidad”. José Enrique Rodó. Ariel. Belén Castro Morales edición. Madrid: Anaya/Mario Muchnik, 1995. 125-161.

. "Introducción". José Enrique Rodó. Ariel. Belén Castro edición. Madrid: Ediciones Cátedra, 2000. 9-128.

Deleuze, Gilles y Felix GuatTari. Rhizome. Paris: Les Editions de Minuit, 1976.

DesCOMBES, VincENT. Das Selbst und das Andere. Fünfundvierzig Jabre Philosophie in Frankreich: 1933-1978. Frankfurt am Main: Suhrkamp, 1981.

ECO, UMBERTO. Opera aperta. Milano: Bompiani, 1962.

La struttura assente. Milano: Gruppo Editoriale Fabbri/Bompiani, 1968. 
ETTE, OTTMAR. "Lateinamerika und Europa. Ein literarischer Dialog und seine Vorgeschichte". José Enrique Rodó. Ariel. Traducción y edición Ottmar Ette. Mainz: Dieterich'sche Verlagsbuchhandlung, 1994. 9-58. jekt einer hispanoamerikanischen Moderne”. José Enrique Rodó. Ariel. Traducción y edición Ottmar Ette. Mainz: Dieterich'sche Verlagsbuchhandlung, 1994. 193-240.

"Así habló Próspero. Nietzsche, Rodó y la modernidad filosófica de 'Ariel'". Cuadernos Hispanoamericanos (Madrid) 528 (junio 1994): 48-62.

-."Literaturwissenschaft als Lebenswissenschaft. Eine Programmschrift im Jahr der Geisteswissenschaften". Lendemains (Tübingen) 32-125 (2007): 7-32.

ETTE, OTTMAR (ed.). Nanophilologie. Literarische Kurz- und Kürzestformen in der Romania. Tübingen: Max Niemeyer Verlag, 2008.

- Literatura en movimiento. Espacio y dinámica de una escritura transgresora de fronteras en Europa y América Latina. Madrid: CSIC, 2009.

Alexander von Humboldt und die Globalisierung. Das Mobile des Wissens. Frankfurt am Main/Leipzig: Insel Verlag, 2009.

2010.

ÜberLebensWissen I-III. Berlin: Kulturverlag Kadmos, [2004]

Konvivenz. Literatur und Leben nach dem Paradies. Berlin: Kulturverlag Kadmos, 2012.

"Weiter denken. Viellogisches denken / viellogisches Denken und die Wege zu einer Epistemologie der Erweiterung". Romanistische Zeitschrift für Literaturgeschichte / Cabiers d'Histoire des Littératures Romanes (Heidelberg) 40.1 .4 (2016): 331-355.

WeltFraktale. Wege durch die Literaturen der Welt. StuttgartWeimar: J.B. Metzler, 2017.

y Titus HeYDEnReich (eds.). José Enrique Rodó y su tiempo. Cien años de "Ariel". Frankfurt am Main/Madrid: Vervuert/Iberoamericana, 2000. 
y SERgio Ugalde QuinTANa (eds.). La filología como ciencia de la vida. México: Universidad Iberoamericana, 2015.

García Morales, AlFONSO. Literatura y pensamiento hispánico de fin de siglo: Clarín y Rodó. Sevilla: Secretariado de Publicaciones, 1992. 75-88. González EChevarría, RoBerTo. "The Case of the Speaking Statue: 'Ariel' and the Magisterial Rhetoric of the Latin American Essay". The Voice of the Masters. Writing and Authority in Modern Latin American Literature. Austin: University of Texas Press, 1985. 8-32.

GuTIÉRREZ GIRARDOT, RAFAEL. "La literatura hispanoamericana de fin de siglo". Luis Iñigo Madrigal (ed.). Historia de la literatura hispanoamericana. Tomo II: Del neoclasicismo al modernismo. Madrid: Cátedra, 1987. 495-506.

Humboldt, AleXANDer Von. Briefe von Alexander von Humboldt an Varnhagen von Ense aus den Jabren 1827 bis 1858. Nebst Auszügen aus Varnhagen's Tagebüchern und Briefen von Varnhagen und Andern an Humboldt. Edición Ludmilla Assing. Leipzig: F.A. Brockhaus, 1860.

"Anregungsmittel zum Naturstudium. Reflex der Außenwelt auf die Einbildungskraft: Dichterische Naturbeschreibung. Landschaftsmalerei. Cultur exotischer Gewächse, den physiognomischen Charakter der Pflanzendecke auf der Erdoberfläche bezeichnend". Kosmos. Entwurf einer physischen Weltbeschreibung. Frankfurt am Main: Eichborn Verlag (Die Andere Bibliothek), 2004. 189-239.

IBÁÑEZ, RoBerTo. "El ciclo de Proteo". Cuadernos de Marcha (Montevideo) 1 (mayo de 1967): 7-53.

KRISTEVA, Julia. Étrangers à nous-mêmes. Paris: Gallimard, 1991.

Menke, CHRistopH. Kraft. Ein Grundbegriffästhetischer Anthropologie. Frankfurt am Main: Suhrkamp, 2008.

Meyer-Minnemann, Klaus. Der spanisch-amerikanische Roman des Fin de siècle. Tübingen: Niemeyer, 1979.

Real de AzÚA, CARlos. "Prólogo a Motivos de Proteo". José Enrique Rodó. Ariel. Motivos de Proteo. Ángel Rama edición y cronología. Caracas: Biblioteca Ayacucho, 1976. ix-xxxv. 
. "Prólogo". José Enrique Rodó. Motivos de Proteo. Montevideo: Biblioteca Artigas, 1957. vii-clviii.

"Rodó en sus papeles. A propósito de la exposición". Escritura (Montevideo) (3 de marzo de 1948): 89-103.

Rodó, José EnRique. Obras completas. Emir Rodríguez Monegal edición, introducción y notas. Madrid: Aguilar, 1967.

Rodríguez Monegal, Emir. "Introducción general", "Prólogo [a Motivos de Proteo]". José Enrique Rodó. Obras completas. Madrid: Aguilar, 1967. 19-139, 301-308.

RUEDA, ANA. Minificción y nanofilología. Latitudes de la hiperbrevedad. Madrid-Frankfurt am Main: Iberoamericana/Vervuert, 2017.

Simanowski, ROBERTO. Facebook-Gesellschaft. Berlin: Matthes \& Seitz, 2016.

SuIfFET, Norma. José Enrique Rodó. Su vida, su obra, su pensamiento. Montevideo: Ediciones La Urpila, 1995.

TODOROV, TZVETAN. La conquête de l'Amérique. La question de l'autre. Paris: Les Editions du Seuil, 1982. 